\title{
Therapeutic Experience on Stance Control Knee- Ankle-Foot Orthosis With Electromagnetically Controlled Knee Joint System in Poliomyelitis
}

\author{
Jung-Hwan Kim, MD ${ }^{1}$, Sang-Goo Ji, PhD², Kang-Jae Jung, MD ${ }^{1}$, Jae-Hyung Kim, MD, PhD \\ Departments of ${ }^{1}$ Physical Medicine and Rehabilitation and ${ }^{2}$ Physical Therapy, \\ Eulji University Hospital \& Eulji University School of Medicine, Daejeon, Korea
}

\begin{abstract}
A 54-year-old man with poliomyelitis had been using a conventional, passive knee-ankle-foot orthosis (KAFO) with a drop ring lock knee joint for about 40 years. A stance control KAFO (SCKAFO) with an electromagnetically controlled (E-MAG) knee joint system was prescribed. To correct his gait pattern, he also underwent rehabilitation therapy, which included muscle re-education, neuromuscular electrical stimulation, strengthening exercises for the lower extremities, and balance training twice a week for about 4 months. Both before and after rehabilitation, we conducted a gait analysis and assessed the physiological cost index in energy expended during walking in a locked-knee state and while he wore a SCKAFO with E-MAG. When compared with the pre-rehabilitation data, the velocity, step length, stride length, and knee kinematic data were improved after rehabilitation. Although the SCKAFO with E-MAG system facilitated the control of knee motion during ambulation, appropriate rehabilitative therapy was also needed to achieve a normal gait pattern.
\end{abstract}

Keywords Rehabilitation, Poliomyelitis, Orthotic devices

\section{INTRODUCTION}

Weakness of the knee extensor muscles can be caused by neurological diseases and muscular diseases, such as post-polio syndrome, spinal cord injury, trauma, multiple sclerosis, muscular dystrophy, unilateral leg paraly-

Received May 26, 2015; Accepted July 20, 2015

Corresponding author: Jae-Hyung Kim

Department of Physical Medicine and Rehabilitation, Eulji University Hospital and Eulji University School of Medicine, 95 Dunsanseo-ro, Seogu, Daejeon 35233, Korea

Tel: +82-42-611-3629, Fax: +82-42 611-3633, E-mail: rehabkjh@hanmail. net

(c) This is an open-access article distributed under the terms of the Creative Commons Attribution Non-Commercial License (http://creativecommons. org/licenses/by-nc/4.0) which permits unrestricted noncommercial use, distribution, and reproduction in any medium, provided the original work is properly cited.

Copyright $\odot 2016$ by Korean Academy of Rehabilitation Medicine sis, and paresis [1]. Such weakness can result in a labored and unsafe gait, which causes fatigue and a slow walking speed. For persons with weakness of the quadriceps muscle, a knee-ankle-foot orthosis (KAFO) is often prescribed when the muscles that control the knee joint are weakened.

A SCKAFO locks the knee joint during the stance phase and allows free rotations during the swing phase of ambulation [2]. By allowing the knee to bend during the swing phase, the SCKAFO provides a much more normal gait than the gait achieved with the conventional, passive KAFO, so patients are able to walk with less effort, and gait compensation may be reduced. Electromagnetic (E-MAG) knee joint systems combine mechanics and electronics in order to maximize mobility, safety, and reliability and are the first such systems to have an electro- 
magnetic lock operated by an electronic control unit.

To date, no clinical studies of the SCKAFO with E-MAG system have been carried out. In this case report, we describe a patient with poliomyelitis who received the KAFO with E-MAG system, and we present the results of his rehabilitation with regard to gait parameters, kinematic data, and the energy expenditure of walking.

\section{CASE REPORT}

The patient was a 54-year-old man who had contracted poliomyelitis at a young age. He was completely ambulatory, having used a left-sided conventional, passive KAFO with a drop ring lock knee joint for about 40 years. He had no history of falls or bone fractures. Function of both upper limbs was normal. His left knee and ankle were flaccid and showed muscle atrophy. Based on standard manual muscle testing (MMT) [3], the grades for his left hip strength were extension $2 / 5$, abduction $2 / 5$, flexion $2 / 5$, and adduction $2 / 5$; left knee strength grades were extension $1 / 5$ and flexion $2 / 5$; and left ankle strength grades were dorsiflexion $2 / 5$ and plantarflexion $2 / 5$. The left hip was capable of a full passive range of motion, and the passive ranges of motion for the left knee were $150^{\circ}$ of flexion and $-20^{\circ}$ of extension. His left leg was shorter than his right by $2.5 \mathrm{~cm}$. The muscle strength of right hip, knee, and ankle was $4 / 5$. Pes cavus and claw toe deformities were evident in his left foot. Passive ranges of motion of the left ankle were $10^{\circ}$ of dorsiflexion and $35^{\circ}$ of plantarflexion. His gait pattern reflected overuse of the left pelvic girdle muscles, and left pelvic hiking and circumduction resulted in sufficient toe clearance during the swing phase. On the left side, severe genu recurvatum was observed during the stance phase. We prescribed a SCKAFO with E-MAG controlled knee joint system (17B200 E-MAG control joint; Otto Bock, Duderstadt, Germany) as well as rehabilitation therapy to correct his gait pattern, which consisted of muscle re-education of the pelvis-hip-knee-ankle components, neuromuscular electrical stimulation therapy, strengthening exercises for the lower extremities, and balance training twice a week for 4 months. We performed gait analysis with the knee in a locked state and before and after rehabilitative therapy at the motion analysis research center.

\section{Temporospatial data and kinematic data}

During the gait evaluation, the kinematic data were acquired with eight Eagle Digital RealTime cameras (Motion Analysis Corp., Santa Rosa, CA, USA) and were processed with OrthoTrak (Motion Analysis Corp.). We used MATLAB (MathWorks Inc., Natick, MA, USA) and Excel (Microsoft Corp., Redmond, WA, USA) to calculate temporospatial data and generate kinematic graphs. Table 1 presents the mean ( \pm standard deviation) values for the temporospatial parameters for this patient. Before rehabilitation, walking with a SCKAFO with E-MAG resulted in a mean slower walking speed (velocity) and a longer stride length compared with walking with the knee locked, which may be attributed to poor adaptation to the new orthosis and poor balance. After rehabilitation

Table 1. Temporospatial data for 54-year-old man wearing SCKAFO with E-MAG controlled knee joint system

\begin{tabular}{|c|c|c|c|}
\hline \multirow{2}{*}{ Parameter } & \multirow{2}{*}{ Locked-knee state } & \multicolumn{2}{|c|}{ SCKAFO with E-MAG } \\
\hline & & Pre-rehabilitation & Post-rehabilitation \\
\hline Velocity $(\mathrm{cm} / \mathrm{s})$ & $47.1(2.31)$ & $37.5(6.11)$ & $79.5(2.11)$ \\
\hline Cadence (steps/min) & $65.7(2.07)$ & $52.3(3.17)$ & $80.8(2.23)$ \\
\hline Stride length $(\mathrm{cm})$ & $86.1(2.53)$ & $87.5(8.29)$ & $118.8(2.74)$ \\
\hline Step width $(\mathrm{cm})$ & $17.5(0.75)$ & $17.0(0.43)$ & $14.4(1.35)$ \\
\hline Left step length $(\mathrm{cm})$ & $35.8(1.57)$ & $36.1(6.29)$ & $49.9(2.16)$ \\
\hline Left stance (\% cycle) & $63.0(1.67)$ & $69.8(3.35)$ & $55.5(3.95)$ \\
\hline Left swing (\% cycle) & $37.0(1.67)$ & $30.2(3.35)$ & $44.5(3.95)$ \\
\hline Right step length (cm) & $50.2(1.54)$ & $51.0(3.66)$ & $68.6(1.87)$ \\
\hline Right stance (\% cycle) & $72.1(1.06)$ & $76.0(1.72)$ & $69.5(1.11)$ \\
\hline Right swing (\% cycle) & $27.9(1.06)$ & $24.0(1.72)$ & $30.5(1.11)$ \\
\hline
\end{tabular}

Values are presented as mean (standard deviation).

SCKAFO, stance control knee-ankle-foot orthosis; E-MAG, electromagnetic. 
with the SCKAFO with E-MAG, the mean velocity and cadence were faster than those measured before rehabilitation. The post-rehabilitation step length and stride length were longer than the pre-rehabilitation values. Before rehabilitation, walking with a SCKAFO with E-MAG resulted in a longer stance phase compared with walking with a locked knee. The stance phase length before rehabilitation was shorter than it was after rehabilitation. Thus, rehabilitative therapy with a SCKAFO with E-MAG improved this patient's velocity, cadence, step length, stride length, and step width.

During the mid-swing phase, the SCKAFO maximal
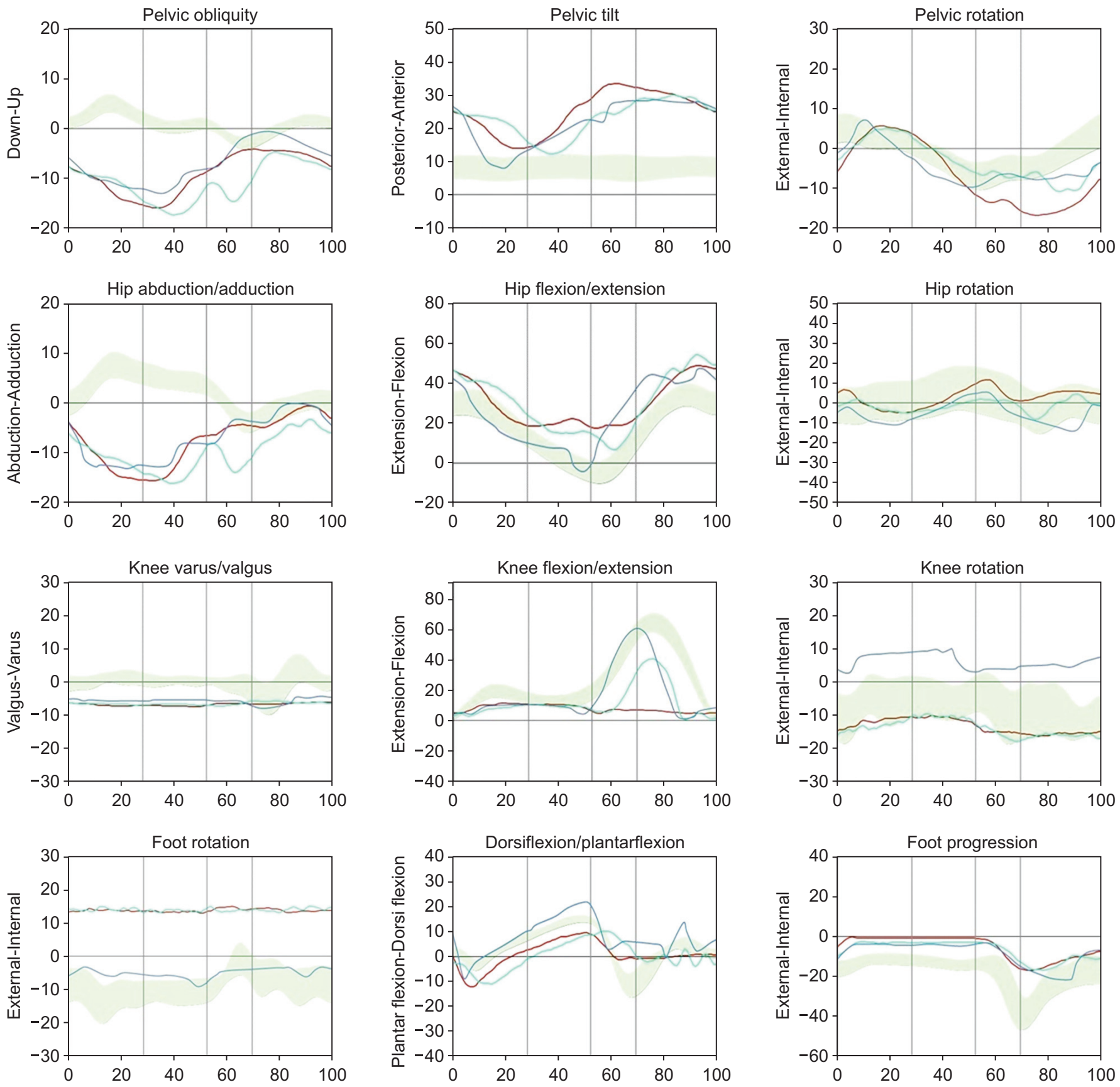

Fig. 1. Kinematic data for left lower extremity using gait analysis for walking while wearing a SCKAFO with E-MAG system (blue line, post-rehabilitation; sky-blue line, pre-rehabilitation; red line, locked-knee state). SCKAFO, stance control knee-ankle-foot orthosis; E-MAG, electromagnetic. 
knee flexion prior to rehabilitation was $39.98^{\circ} \pm 3.07^{\circ}$, as compared with $10.26^{\circ} \pm 3.07^{\circ}$ during walking with a locked knee joint. After rehabilitation, maximal knee flexion was increased to $59.15^{\circ} \pm 2.42^{\circ}$. On gait analysis after rehabilitation, pelvic obliquity, hip abduction/adduction, knee flexion/extension, and foot rotation on the left side were improved, as compared with the pre-rehabilitation data (Fig. 1). Use of both SCKAFO with E-MAG and rehabilitative therapy resulted an overall pattern of knee motion that closely matched that of normal walking.

\section{Energy expenditure of walking}

The physiological cost index (PCI) is a simple measurement of gait efficiency and energy expenditure of walking that is often used to evaluate a prosthesis and orthosis. PCI is calculated as follows: heart rate (bpm) after a 3-minute walk minus heart rate (bpm) at rest divided by speed $(\mathrm{m} / \mathrm{min})[4]$. The PCI for walking wearing a SCKAFO with a locked knee, with E-MAG before rehabilitation, and with E-MAG after rehabilitation were determined based on the heart rates at rest and at 3 minutes after walking and were $0.62,0.57$, and 0.55 , respectively.

\section{DISCUSSION}

The SCKAFO improves gait kinematics, increases knee flexion during the swing phase, provides a more symmetrical gait, and requires less compensatory movement than the conventional, passive KAFO [5-7]. However, there are three major problems with the SCKAFO designs: switching between the stance and swing phases is not smooth, the knee joint is locked during the stance phase, and the device does not assist in knee extension. In order to lock and unlock the knee joint, most SCKAFOs require patients to fully extend their knees, which results in a discontinuous gait and require the patient to attend to changing their gait $[7,8]$. SCKAFO must be designed so that it meets important functional, safety, and cosmetic needs. In the E-MAG control knee joint system, the locked stance phase and the unlocked swing phase are electromagnetically controlled. An intelligent sensor system measures the position of the leg during walking and controls the orthotic joint accordingly. An electronic control unit and remote control are also required in order to operate the joint, with power supplied by a battery (Fig. 2). The system operates the lock and provides feedback regarding the knee joint status, reporting the joint position as well as its operating state. As a result, the patient can rely on the joint in any situation.

The E-MAG system is suitable for patients with higher mobility requirements who were previously unable or unwilling to obtain an adequately fitting orthotic for various anatomical reasons, such as leg-length reduction, valgus and varus deformities of the knee joint, or a severely atrophic leg. An appreciable portion of the population using fixed-knee KAFOs has sufficient hip strength to benefit from a SCKAFO. Prescription criteria typically require hip muscle strength of at least grade 3 [7]. Our patient's hip muscle strength on MMT was less than this; however, we were able to achieve a satisfactory outcome with the SCKAFO with E-MAG after the patient underwent rehabilitation. This result confirmed that even if the hip extensor and flexor muscle strength is less than grade 3, a unilateral SCKAFO with E-MAG can be prescribed if the patient demonstrates reciprocal gait pattern, as was the case here. Hip strength and control requirements may be reduced for a SCKAFO with E-MAG system, suggesting a broad indication for this system in patients with poliomyelitis.

In general, the characteristics of the gait in poliomyelitis are excessive knee extension and inadequate knee flexion, excessive hip flexion during the late swing phase, and excessive plantarflexion during the weight-

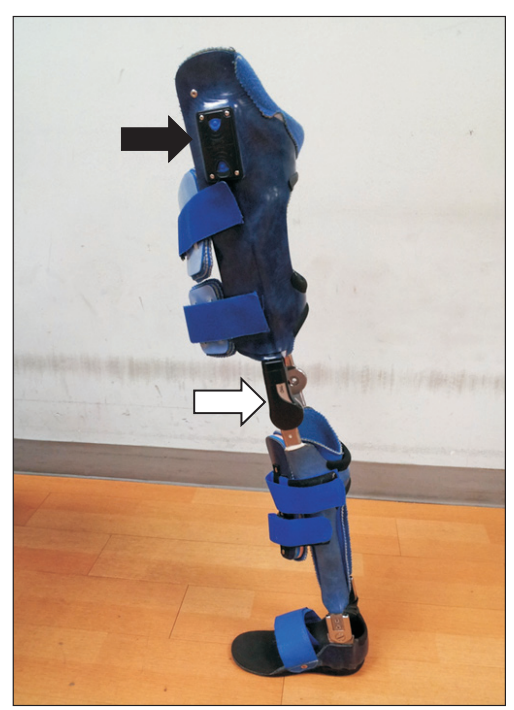

Fig. 2. Stance control knee-ankle-foot orthosis (SCKAFO) with electromagnetic (E-MAG) system (white arrow, EMAG knee joint; black arrow, battery). 
acceptance phases. Our patient exhibited a similar gait pattern; however, in the stance phase, pelvic obliquity to the left and downward as well as hip abduction were also detected in the kinematic data, and we thought these findings were due to chronic pelvic muscle weakness and leg-length discrepancy. After proper rehabilitation, the SCKAFO with E-MAG system improved our patient's gait by eliminating the abnormal pelvic drop during $52 \%$ to $69 \%$ of the gait cycle, and we could observe a decrease in hip abduction when he was walking with the SCKAFO with E-MAG, indicating less circumduction of his braced leg during the swing phase. The effect of knee flexion on the braced limb could be seen directly, with a reduction in pelvic obliquity and hip abduction suggesting a more efficient gait pattern overall. We thought an improvement of gait pattern and a reduction of compensatory motions might be associated with the facilitated pelvic and trunk control ability.

The SCKAFO accommodation period may not have been long enough for this patient to overcome the walking strategies previously applied for conventional KAFO or nondisabled walking. Therefore, accommodation periods of at least 3 months should be considered [9]. Our patient participated in a rehabilitative program that included muscle re-education, neuromuscular electrical stimulation therapy, the strengthening exercises for the lower extremities, and balance training for 4 months. The rehabilitative therapy was focused on pelvic and trunk control and gait training. Although there was no significant change in muscle strength on MMT after rehabilitation, the patient did express his satisfaction with the result. When a SCKAFO with E-MAG is prescribed to a patient with poliomyelitis, it is important to institute a proper rehabilitation program.

The PCI has been used to study the gait performance of normal individuals, children, and person with poliomyelitis because it correlates reasonably well with oxygen uptake [10]. Our results are consistent with the findings in other poliomyelitis patients. Compared with a locked knee, use of a SCKAFO with E-MAG appears to improve energy efficiency.

Our study had some limitations. First, we could not perform a complete kinetic study, so our results are limited to kinematic data obtained on gait analysis. If a kinetic analysis had been performed, we could have obtained more information from the gait analysis. Second, we could not explain whether the improvement noted on gait analysis was an orthotic effect or a rehabilitative effect. Nevertheless, on the basis of our results, we suggest that appropriate rehabilitative therapy combined with a SCKAFO with E-MAG can improve gait parameters on walking as well as energy efficiency for the patient with poliomyelitis.

\section{CONFLICT OF INTEREST}

No potential conflict of interest relevant to this article was reported.

\section{ACKNOWLEDGMENTS}

This research was supported by grants from the Eulji Medi-Bio Research Institute (EMBRI), Eulji University in Daejeon, Korea (No. 2013-EMBRI-DJ0003).

\section{REFERENCES}

1. Cullell A, Moreno JC, Rocon E, Forner-Cordero A, Pons JL. Biologically based design of an actuator system for a knee-ankle-foot orthosis. Mech Mach Theory 2009;44:860-72.

2. Tian F, Hefzy MS, Elahinia M. State of the art review of knee-ankle-foot orthoses. Ann Biomed Eng 2015;43: 427-41.

3. Hislop H, Montgomery J. Daniels and Worthingham's muscle testing: techniques of manual examination. 7th ed. Philadelphia: Saunders; 2002.

4. Jaiyesimi AO, Fashakin OG. Reliability of physiological cost index measurements. Afr J Med Med Sci 2007; 36:229-34.

5. Arazpour M, Chitsazan A, Bani MA, Rouhi G, Ghomshe FT, Hutchins SW. The effect of a knee ankle foot orthosis incorporating an active knee mechanism on gait of a person with poliomyelitis. Prosthet Orthot Int 2013;37:411-4.

6. McMillan AG, Kendrick K, Michael JW, Aronson J, Horton GW. Preliminary evidence for effectiveness of a stance control orthosis. J Prosthet Orthot 2004;16:613.

7. Yakimovich T, Lemaire ED, Kofman J. Engineering design review of stance-control knee-ankle-foot orthoses. J Rehabil Res Dev 2009;46:257-67. 
8. Shamaei K, Napolitano PC, Dollar AM. Design and functional evaluation of a quasi-passive compliant stance control knee-ankle-foot orthosis. IEEE Trans Neural Syst Rehabil Eng 2014;22:258-68.

9. Irby SE, Bernhardt KA, Kaufman KR. Gait changes over time in stance control orthosis users. Prosthet Orthot Int 2007;31:353-61.
10. Hachisuka K, Makino K, Wada F, Saeki S, Yoshimoto N. Oxygen consumption, oxygen cost and physiological cost index in polio survivors: a comparison of walking without orthosis, with an ordinary or a carbon-fibre reinforced plastic knee-ankle-foot orthosis. J Rehabil Med 2007;39:646-50. 